\title{
Polymorphism R92Q of the tumour necrosis factor receptor 1 gene is associated with myocardial infarction and carotid intima-media thickness - The ECTIM, AXA, EVA and GENIC Studies
}

\author{
Odette Poirier ${ }^{1}$, Viviane Nicaud ${ }^{1}$, Jérôme Gariépy $^{2}$, Dominique Courbon ${ }^{3}$, Alexis Elbaz ${ }^{4}$, \\ Caroline Morrison $^{5}$, Franck Kee ${ }^{6}$, Alun Evans ${ }^{6}$, Dominique Arveiler ${ }^{7}$, Pierre Ducimetière ${ }^{3}$, \\ Pierre Amarenco ${ }^{8}$, François Cambien*,1

\begin{abstract}
${ }^{1}$ INSERM U525, Epidemiologic and Molecular Genetics of Cardiovascular Diseases, Paris, France; ${ }^{2}$ Department of Prevention of Cardiovascular Diseases, Georges Pompidou European Hospital, Paris, France; ${ }^{3}$ INSERM U258, Cardiovascular and Metabolic Epidemiology, Villejuif, France; ${ }^{4}$ INSERM U360, Epidemiologic Researches in Neurology and Psychopathology, Paris, France; ${ }^{5}$ MONICA Register, Glasgow Health Board, Glasgow, UK; ${ }^{6}$ Department of Epidemiology and Public Health, Queen's University, Belfast, UK; ${ }^{7}$ MONICA Register, Laboratory of Epidemiology and Public Health, Strasbourg, France; ${ }^{8}$ Department of Neurology, Lariboisière Hospital, Paris, France
\end{abstract}

The TNFRS1A gene was screened for polymorphisms in 95 subjects with premature myocardial infarction (MI), who also had one parent who had an MI. A total of 10 polymorphisms were found: three in the promoter region, two in exons and five in introns. All polymorphisms were genotyped in ECTIM, a case-control study of $\mathrm{MI}$ (1815 subjects). The nonsynonymous $92 \mathrm{Q}$ allele was found in $1.8,1.0$ and $1.7 \%$ of controls from Strasbourg, Belfast and Glasgow - respectively; in cases: 4.2, 2.2 and 3.2\%. The populationadjusted odds ratio (OR) for $\mathrm{MI}$ associated with allele $\mathrm{Q}$ carrying was 2.15 (95\% $\mathrm{Cl}$ : $1.09-4.23)$. To check its possible implication in atherosclerosis, this polymorphism was then genotyped in the AXA Study (ultrasound examinations of carotid and femoral arteries in the context of an employment medical examination, 733 subjects), the EVA Study (ultrasound examinations of carotid arteries in a study of cognitive and vascular ageing, 1092 subjects) and the GENIC Study (on brain infarction (BI), 912 subjects). In the AXA Study, among smokers, carrying the $92 \mathrm{Q}$ allele was positively associated with the presence of a carotid plaque (OR 5.07; $95 \% \mathrm{Cl}: 1.64-15.63$ ) and with a thickening of the carotid intima-media thickness (IMT) $(0.59(0.11)$ vs $0.54(0.11), P=0.045)$. In the EVA Study, carriers of allele $92 Q$ had an increased mean carotid IMT $(0.70(0.09)$ vs $0.67(0.13), P=0.02)$. No significant association of the $92 \mathrm{Q}$ allele was found with $\mathrm{BI}$ in the GENIC Study. Overall, these results may suggest that carriers of the $92 \mathrm{Q}$ allele may be at increased risk of atherosclerosis.

European Journal of Human Genetics (2004) 12, 213-219. doi:10.1038/sj.ejhg.5201143

Published online 24 December 2003

Keywords: TNFRSF1A; genetic polymorphisms; atherosclerosis

*Correspondence: Dr F Cambien, INSERM U525, Epidemiologic and Molecular Genetics of Cardiovascular Diseases, Faculté de Médecine Pitié-Salpêtrière, 91 Boulevard de l'Hôpital, 75634 Paris cedex 13, France. Tel: + 331407796 39; Fax: 331407797 28;

E-mail: francois.cambien@chups.jussieu.fr

Received 27 August 2003; revised 7 November 2003; accepted 14 November 2003
Introduction

A number of arguments support the role of tumour necrosis factor (TNF)- $\alpha$, in the initiation, development and complications of atherosclerosis. This multifunctional proinflammatory cytokine is expressed in endothelial and 
smooth muscle cells at all stages of plaque formation. ${ }^{1}$ It renders the local endothelium prothrombotic and proinflammatory, ${ }^{2,3}$ stimulates the expression of adhesion molecules such as vascular cell adhesion molecule 1 (VCAM1), and favours the recruitment of inflammatory cells. ${ }^{4}$ It stimulates the expression of tissue factor and the release of interleukin-1, nitric oxide and a number of other mediators that are important in atherosclerosis. It also activates matrix metalloproteinases and may be involved in vascular remodelling and plaque destabilization.

We have previously shown that a polymorphism of $T N F-\alpha$ was present at a higher frequency in Belfast, a highrisk population for myocardial infarction (MI), compared to France, and was associated with parental MI, but not with the presence of MI in patients participating in the ECTIM Study. ${ }^{5}$ TNF- $\alpha$ binds to two receptors on the cell surface: p75 and p55. Our previous result prompted us to screen the p55 receptor gene (TNF receptor superfamily, member 1A TNFRSF1A) for polymorphisms, because it has been shown that endothelial cell responses to TNF- $\alpha$, such as expression of tissue factor and adhesion molecules by mononuclear cells (which may be important in the pathogenesis of atherosclerosis) are mediated predominantly by this receptor. $^{6}$ We looked for associations between the identified polymorphisms and MI in the ECTIM Study. Owing to the positive association found with one of these, we studied this polymorphism in other studies, in relation to brain infarction (BI) and measures of carotid and femoral atherosclerotic plaques and intima-media thickness (IMT).

\section{Methods \\ Studies}

ECTIM The aim of the ECTIM Study was to identify genetic polymorphisms affecting the susceptibility to $\mathrm{MI}$ in patients and controls from the United Kingdom and France. Its design was described in 1992. ${ }^{7}$ Men aged 25-64 years were recruited between 1989 and 1991 from four WHO/ MONICA (MONItoring trends and determinants in CArdiovascular diseases) centres, in Northern Ireland (Belfast) and France (Lille, Strasbourg, Toulouse). In 1998, the study population has been modified owing to exhaustion of DNA from two recruitment centres (Lille and Toulouse, France) and extensions of the recruitment in Belfast and Glasgow (United Kingdom). ${ }^{8}$ The results reported here are based on samples of Strasbourg (France), Belfast (Northern Ireland) and Glasgow (Scotland). The two centres in the United Kingdom recruited both men and women, whereas only men were recruited in France. Cases $(n=1107)$ aged 25-64 years at MI for men and 25-69 years for women were recruited between 3 and 9 months ( 2 years for women in Belfast) after the index MI. Controls $(n=1082)$ of comparable age and sex were recruited from the lists of general practitioners in the same areas in the United Kingdom, and from the electoral rolls in France. Their parents had to be born in this same region and their four grandparents had to have been born in Europe. Genetic informed consent was obtained from all subjects. The mean age of cases with MI was 56.1 years (range 28-72), and the mean age of controls was 56.5 (range $27-71$ ).

$\boldsymbol{A X A}$ The aim of this study was to identify genetic factors that may affect Doppler-assessed parameters of the carotid and femoral arteries in healthy individuals; 777 subjects were recruited in the context of an employment medical examination (insurance company). ${ }^{9}$ The mean age was 42.7 years (range 17-64). Ethnic origin was not an exclusion criterion. However, $95 \%$ of the subjects were of European ancestry. The study protocol was approved by the Ethics Committee, and written consent was obtained from all participants. All subjects underwent carotid and femoral Doppler examination, designed to quantify the IMT and the degree of arteriosclerosis and calcification in these two sites. The excellent quality of the measurements allowed evaluation of atherosclerosis and plaque calcifications in unselected apparently 'healthy' subjects, which obviously cannot be achieved by angiography. ${ }^{10}$ The average IMT was calculated as the mean value of a great number of local IMT measures. For each subject, a mean IMT (left + right/2) was taken as a measure of current wall thickness. A thickness $\geqslant 2 \mathrm{~mm}$ was considered to be plaque.

EVA Details of the EVA Study have been reported previously. ${ }^{11}$ It is a longitudinal study of cognitive and vascular ageing. The study population is composed of volunteers aged 59-71 years, who were recruited from the electoral rolls of the city of Nantes (western France). Among the 1092 subjects genotyped for R92Q, 97\% were born in Metropolitan France and had both parents born in Europe. The study protocol was approved by the Ethics Committee, and written informed consent was obtained from all subjects. During the baseline visit, which took place between January 1992 and July 1993, the subjects had high-resolution ultrasound examinations of the carotid arteries. The mean age of subjects was 64.2 years (range 59-71). Both the near and far wall of common carotid arteries (CCA), the carotid bifurcations and the origins (first $2 \mathrm{~cm}$ ) of the internal carotid artery were scanned longitudinally and transversally to assess the presence of plaques, which were defined as localized echostructures that encroached into the vessel of more than $1 \mathrm{~mm}$ beyond the interface between the lumen and intima. ${ }^{12}$ Subjects had 100 IMT measures automatically performed at the far wall of both CCAs at a site free of plaque. The mean of the measures was calculated at each site and the mean of the two sites was studied.

GENIC The GENIC Study is a case-control study of genetic susceptibility to BI, which involved 12 French hospital neurological departments. Details of the protocol 
have been reported elsewhere. ${ }^{13}$ Cases were consecutively recruited in the week following the event if they had clinical symptoms suggestive of stroke and if an infarct was proven by magnetic resonance imaging. Cases were classified into aetiologic subtypes of BI (ie, atherothrombotic, cardioembolic, lacunar, arterial dissections, rare aetiologies, unknown or undetermined cause) according to prespecified criteria, by two neurologists. Controls without a history of stroke were recruited among individuals hospitalized at the same institutions for a reason other than neurological or acute myocardial disease. One control was matched by sex, age ( \pm 5 years) and centre to each case. Parents of both cases and controls had to be of European descent. The research protocol was approved by the Ethics Committee, and all subjects signed an informed consent. A total of 510 cases and the same number of controls were recruited. The mean age of cases with BI was 66.2 years (range 20-85), and the mean age of controls was 66.1 (range 20-89). The protocol for scanning of the carotid arteries in cases and controls was the same as in the EVA Study.

\section{Identification of polymorphisms}

In all, 95 subjects with MI (below 60 years in males and 65 years in females), whose parents had also had an MI, were selected from the ECTIM Study and their genomic DNA was screened by SSCP for TNFRSF1A polymorphisms. From the published sequences ${ }^{14,15}$ (GENBANK NID: 339746 and 288493), 16 PCR fragments were generated. In all, $596 \mathrm{bp}$ were screened in the $5^{\prime}$ region, $211 \mathrm{bp}$ in the $5^{\prime}$ UTR region, $1368 \mathrm{bp}$ in the coding sequence, $1056 \mathrm{bp}$ in the intronic regions flanking exons and $541 \mathrm{bp}$ in the $3^{\prime}$ UTR region. SSCP analysis, sequencing and genotyping using allelespecific oligonucleotides (ASO) were performed as previously described. ${ }^{16}$ The PCR primers, conditions used for amplifying the regions encompassing the polymorphic sites and ASOs may be found on our web site: http:// www.genecanvas.org

\section{Statistical analysis}

Data were analysed using the SAS package. The HardyWeinberg equilibrium was tested by a $\chi^{2}$ test ( 1 DF) separately in cases and controls.

ECTIM Allele frequencies were calculated from genotype frequencies by allele counting. The genotype and allele frequencies were compared between cases and controls, stratified by centre and after the exclusion of controls with coronary heart disease. For the R92Q polymorphism, the odds ratio (OR) for MI associated with the presence of allele $\mathrm{Q}$ was calculated by logistic regression adjusted on age, sex and centre. Heterogeneity of the ORs between centres, between males and females, below and above the median age and between smokers and non-smokers was tested by introducing the corresponding interaction term in the regression model (1 DF).
$\boldsymbol{A X} \boldsymbol{A}$ The association between the $\mathrm{R} 92 \mathrm{Q}$ polymorphism and the presence of at least one carotid plaque $\geqslant 2 \mathrm{~mm}$ was tested in the whole sample and in smokers by a logistic regression procedure. The mean IMT of the right and left CCA, as well as the mean of these two values, was compared between RR and RQ carriers in the whole sample and separately in smokers (current or ex-smokers) and never smokers by a general linear model procedure. Owing to small numbers, genotypic means were compared by a Wilcoxon rank-sum test.

$\boldsymbol{E} \boldsymbol{V A}$ The prevalence of at least one carotid plaque $>1 \mathrm{~mm}$ was compared between carriers of the $\mathrm{Q}$ allele and $\mathrm{RR}$ homozygous carriers in the whole sample and separately in smokers and nonsmokers by a logistic regression procedure. The mean IMT of the CCAs was compared across genotypes in the whole sample and separately in smokers (current and ex-smokers) and never smokers by a general linear model. Owing to low numbers, genotypic means were compared by a Wilcoxon rank-sum test.

GENIC The genotype distribution was compared between cases and controls using conditional logistic regression analysis for matched sets. ${ }^{17}$ The relation between BI and the genotype was studied overall and in each of the four main BI subtypes (atherothrombotic, lacunar, cardioembolic, unknown cause). The relation between carotid plaques and the genotype was studied separately in cases and controls using the Mantel-Haenszel statistic while adjusting for age and sex. The relation between the mean (SEM) IMT of the common carotids and the genotype was studied separately in cases and controls using analysis of covariance.

\section{Results \\ ECTIM}

A total of 10 polymorphisms were detected by screening the TNFRSF1A gene (Table 1): three in the $5^{\prime}$ region (G609T, G-580A, A-393C), two in exons (exon 1, P12P; exon $4, \mathrm{R} 92 \mathrm{Q})$ and five in introns. Five variants were rare $(\leqslant 1 \%)$ and two frequent polymorphisms were tightly associated (G-609T and $C+186 /$ in4T). For details on linkage disequilibrium and haplotypes, see our web site http://www.genecanvas.org

The numbers of genotyped cases and controls were 967 and 836, respectively. There was no significant deviation from Hardy-Weinberg equilibrium for any genotype in any subgroup of cases or controls from each centre. A case/ control difference was found in the frequency of the R92Q polymorphism: carriers of the $92 \mathrm{Q}$ allele were twice as frequent in cases compared to controls and this was seen in all three centres (population-adjusted OR: 2.15, 95\% CI: 1.09-4.23, $P=0.027$; Table 2). Only one 92QQ homozygote (a case) was found and was pooled with hetero- 
Table 1 Description of the polymorphisms of TNFRSF1A (position is indicated from the start of translation) and rare allele frequency in control subjects of the ECTIM Study

\begin{tabular}{lccr}
\hline Polymorphism & Position & Type of variation & Minor allele frequency \\
\hline G-609T & $5^{\prime}$ & $\mathrm{G} / \mathrm{t}$ & 0.40 \\
G-580A & $5^{\prime}$ & $\mathrm{G} / \mathrm{a}$ & $<0.01$ \\
A-383C & $5^{\prime}$ & $\mathrm{A} / \mathrm{c}$ & $<0.01$ \\
P12P & $\mathrm{A} / \mathrm{g}$ & 0.46 \\
G+212/in2 (212th nucleotide of intron 2) & Intron 2 & $<01$ \\
R92Q & Exon 4 & $\mathrm{G} / \mathrm{a}$ & $<0.01$ \\
G+147/in4 (147th nucleotide of intron 4) & Intron 4 & 0.01 \\
C+186/in4T (186th nucleotide of intron 4) & Intron 4 & 0.40 \\
G+10/in6A (10th nucleotide of intron 6) & Intron 6 & 0.42 \\
T+294/in7C (294th nucleotide of intron 7) & Intron 7 & $\mathrm{G} / \mathrm{a}$ & 0.19 \\
\hline
\end{tabular}

Polymorphisms closely associated: G-609T and C+186/in4T (11\% of recombinant haplotypes).

Table 2 Genotype and allele frequency of TNFRSF1A/ $R 92 Q$ in patients with myocardial infarction and controls of the ECTIM Study

\begin{tabular}{|c|c|c|c|c|}
\hline & $R R$ & $R Q$ & QQ & $f(Q)$ \\
\hline \multicolumn{5}{|c|}{ Glasgow } \\
\hline$n$ & 359 & 12 & 1 & \\
\hline$\%$ & 96.5 & 3.2 & 0.3 & 0.019 \\
\hline \multicolumn{5}{|c|}{ Controls } \\
\hline$n$ & 291 & 5 & 0 & \\
\hline$\%$ & 98.3 & 1.7 & 0.0 & 0.008 \\
\hline \multicolumn{5}{|l|}{ Belfast } \\
\hline \multicolumn{5}{|l|}{$\mathrm{MI}$} \\
\hline$n$ & 398 & 9 & 0 & \\
\hline$\%$ & 97.8 & 2.2 & 0.0 & 0.011 \\
\hline \multicolumn{5}{|c|}{ Controls } \\
\hline$n$ & 378 & 4 & 0 & \\
\hline$\%$ & 99.0 & 1.0 & 0.0 & 0.005 \\
\hline \multicolumn{5}{|c|}{ Strasbourg } \\
\hline MI & & & & \\
\hline$n$ & 185 & 8 & 0 & \\
\hline$\%$ & 95.8 & 4.2 & 0.0 & 0.021 \\
\hline \multicolumn{5}{|c|}{ Controls } \\
\hline$n$ & 162 & 3 & 0 & \\
\hline$\%$ & 98.2 & 1.8 & 0.0 & 0.009 \\
\hline \multicolumn{5}{|l|}{ ALL } \\
\hline \multicolumn{5}{|l|}{$\mathrm{MI}$} \\
\hline$n$ & 942 & 29 & 1 & \\
\hline \multirow{2}{*}{\multicolumn{5}{|c|}{ Controls }} \\
\hline & & & & \\
\hline$n$ & 831 & 12 & 0 & \\
\hline$\%$ & 98.6 & 1.4 & 0.0 & 0.007 \\
\hline
\end{tabular}

Odds ratio $(95 \% \mathrm{Cl})$ for $\mathrm{Ml}$ associated with allele $\mathrm{Q}$ carrying, adjusted for age, sex and centre: $2.15(1.09-4.23), P=0.027$.

zygotes for analysis. The OR was not significantly heterogeneous between males and females, smokers and nonsmokers, above and below the median age (55 years). There was no significant association of this polymorphism with parental MI. There was no significant effect of R92Q on phenotypes studied in controls such as body mass index, blood lipids or blood pressure. A borderline case/control difference was also found with the polymorphism of intron 7 (in complete negative linkage disequilibrium with R92Q), CC carriers being slightly less numerous in cases than in controls (OR associated with CC genotype: 0.59, 95\% CI: 0.36-0.98, $P=0.040$ - data not shown). The other SNPs were not significantly associated with MI. A haplotype analysis including the five most frequent polymorphisms and R92Q was run using a maximum likelihood method. ${ }^{18}$ It showed that the $92 \mathrm{Q}$ variant was carried on a single haplotype; apart from the association of 92Q with MI, no other haplotypic effect could be detected.

To investigate possible associations between the R92Q polymorphism and other phenotypes associated with atherosclerosis, this polymorphism (but not the other SNPs) was thereafter investigated in the AXA, EVA and GENIC studies.

\section{AXA}

In AXA, 733 subjects were genotyped for the R92Q polymorphism. No homozygote QQ was found. There was no significant deviation of the genotype frequencies from Hardy-Weinberg equilibrium. The frequency of allele $Q$ was 0.018 . There was a nonstatistically significant excess of RQ heterozygotes among subjects with at least one carotid plaque $\geqslant 2 \mathrm{~mm}$ vs those with no plaque, $7.0 \mathrm{vs} 3.3 \%$ (OR: 2.24, 95\% CI: $0.88-5.70, P=0.092$ ). To further investigate this possible association, the sample was divided into smokers and non-smokers. The association between the R92Q polymorphism and carotid plaques was present in smokers only (Table 3), the frequency of RQ heterozygotes being significantly elevated in those with at least one carotid plaque (OR: 5.07, 95\% CI: 1.64-15.63, $P=0.0048$ ). In smokers, the mean IMT of CCAs was also significantly increased in RQ heterozygotes compared to RR subjects: 0.59 (0.11) vs 0.54 (0.11), $P=0.045$ (Table 4). There was no significant association in non-smokers. No association was found between the polymorphism and IMT or plaque in the femoral arteries. 
Table 3 Percentage of TNFRSF1A/R92Q heterozygotes according to the presence of at least one carotid plaque in the AXA Study

\begin{tabular}{|c|c|c|c|c|c|c|}
\hline & \multicolumn{6}{|c|}{$\% R Q$ carriers } \\
\hline & \multicolumn{2}{|c|}{ All subjects } & \multicolumn{2}{|c|}{ Never smokers } & \multicolumn{2}{|c|}{ Ever smokers } \\
\hline & $N$ & $\%$ of $R Q$ & $N$ & $\%$ of $R Q$ & $N$ & $\%$ of $R Q$ \\
\hline No plaque & $\begin{array}{r}647 \\
86\end{array}$ & 3.3 & $\begin{array}{r}309 \\
24\end{array}$ & 4.5 & 338 & 2.1 \\
\hline
\end{tabular}

Odds ratio $(95 \% \mathrm{Cl})$ for at least one carotid plaque, associated with allele $\mathrm{Q}$ carrying: whole sample: $2.24(0.88-5.70), P=0.092 ;$ in smokers: 5.07 (1.64-15.63), $P=0.0048$.

Table 4 Mean (SD) common carotid intima according to TNFRSF1A/R92Q genotype in the AXA and EVA Studies

\begin{tabular}{|c|c|c|c|c|c|c|c|c|}
\hline & \multicolumn{4}{|c|}{ AXA Study (mean age: 43 years) } & \multicolumn{4}{|c|}{ EVA Study (mean age: 64 years) } \\
\hline & Numbers $R R / R Q$ & $R R$ mean $(S D)$ & $R Q$ mean $(S D)$ & Test $t^{\mathrm{a}}$ & Numbers $R R / R Q$ & $R R$ mean $(S D)$ & $R Q$ mean $(S D)$ & Test ${ }^{\mathrm{a}}$ \\
\hline All & $n=706 / 27$ & $0.53(0.10)$ & $0.54(0.11)$ & $P=0.49$ & $n=1058 / 34$ & $0.67(0.13)$ & $0.70(0.09)$ & $P=0.02$ \\
\hline Never smokers & $n=319 / 14$ & $0.52(0.09)$ & $0.50(0.09)$ & $P=0.36$ & $n=616 / 18$ & $0.65(0.11)$ & $0.68(0.07)$ & $P=0.12$ \\
\hline Ever smokers & $n=387 / 13$ & $0.54(0.11)$ & $0.59(0.11)$ & $P=0.045$ & $n=442 / 16$ & $0.69(0.14)$ & $0.74(0.11)$ & $P=0.08$ \\
\hline
\end{tabular}

Wilcoxon rank-sum test.

\section{EVA}

In EVA, 1092 subjects were genotyped for R92Q. Only one QQ homozygote was found and was pooled with heterozygotes for analysis. There was no significant deviation from Hardy-Weinberg equilibrium. The 92Q allele frequency was 0.016 . There was no association of R92Q with the presence of carotid plaques $>1 \mathrm{~mm}$, in the whole sample or in smokers studied separately (data not shown). However, the mean IMT was significantly increased in RQ carriers compared to RR subjects: $0.70(0.09)$ vs $0.67(0.13)$, $P=0.02$ (Table 4 ).

\section{GENIC}

In GENIC, 456 patients with BI and 456 controls were genotyped for R92Q. No homozygote QQ was found. The frequency of the Q allele was 0.022 in controls. There was no significant deviation from Hardy-Weinberg equilibrium in controls or cases. There was no significant difference in R92Q genotype frequency between cases with BI and controls (Table 5), even when subtypes of BI were considered. There was no excess of RQ heterozygotes in subjects with atherosclerotic plaques of the CCAs compared to subjects with no plaque, in cases as well as in controls, and no increase in IMT (data not shown). Current smoking was strongly associated with $\mathrm{BI}$, but since there were only three smokers carrying the RQ genotype among cases and the same number among controls, it was not possible to study the effects of R92Q in smokers separately. In ever smokers, there was no significant association.
Table 5 Frequency of the TNFRSF1A/R92Q polymorphism in cases with brain infarction and controls of the GENIC Study

\begin{tabular}{lcc}
\hline & \multicolumn{2}{c}{$R 92 Q$ genotype } \\
\cline { 2 - 3 } & $R R$ & $R Q$ \\
\hline Cases with BI & & \\
$n$ & 438 & 18 \\
$\%$ & 96.0 & 4.0 \\
Controls & & \\
$n$ & 436 & 20 \\
$\%$ & 95.6 & 4.4 \\
\hline
\end{tabular}

Odds ratio $(95 \% \mathrm{Cl})$ for brain infarction associated with allele $\mathrm{Q}$ carrying: $0.88(0.46-1.70)$, NS.

\section{Discussion}

Analysis of the sequence of the TNF- $\alpha$ receptor gene resulted in the identification of 10 polymorphisms. All polymorphisms were genotyped in a case-control study of patients with MI and one, R92Q, was associated with MI. This rather uncommon polymorphism is located in the fourth exon of the gene and was the only nonsynonymous polymorphism identified. However, given the number of polymorphisms explored, the possibility that this association is spurious cannot be ignored. We hypothesized that the association observed in the ECTIM Study could reflect an association between the R92Q polymorphism and atherosclerosis development. To test this hypothesis, the distribution of the $\mathrm{R} 92 \mathrm{Q}$ genotypes was investigated in three other studies in which phenotypes associated with 
atherosclerosis were investigated; two focused on atherosclerosis or IMT assessed by echography, and one focused on BI. The AXA and EVA Studies provide echographic assessment of the presence of carotid atherosclerotic plaques and of the IMT in regions devoid of plaques. The R92Q polymorphism was associated with the presence of plaques in the AXA Study and with an increased IMT in both studies, especially in smokers, in whom plaques are much more prevalent (Table 3) and IMT is increased (Table 4). Increased IMT is in general considered as an important correlate of atherosclerosis. ${ }^{11,19}$ The lack of association between the R92Q polymorphism and ischaemic stroke in the GENIC Study may be explained by the lower statistical power of the GENIC Study as compared to the ECTIM Study, especially if the association existed only in a subcategory of stroke. This reduced power would, however, be partially compensated by the higher frequency of the 92Q allele in GENIC than in ECTIM. Another possibility for explaining the lack of association between the R92Q polymorphism and $\mathrm{BI}$ is that the pleiotropic pathophysiological implications of TNF- $\alpha$ through TNFRSF1A signalling are different in the tissue and circulation of the brain and heart. In particular, it has been emphasized that TNF- $\alpha$ is involved in several aspects of stroke development with effects that are often contradictory. ${ }^{20}$

We are aware of only one study investigating possible associations between TNFRSF1A gene polymorphisms and atherosclerosis or coronary artery disease (CAD). Allen et $a l^{21}$ investigated the G-609T and $\mathrm{G}+10 /$ in6A polymorphisms in relation to CAD assessed angiographically and found no significant association. We were also unable to detect any association between these polymorphisms and any phenotype examined in the ECTIM Study.

The R92Q polymorphism is located in the fourth exon of the gene and affects the sequence of the extracellular part of the receptor. To evaluate in silico the putative functionality of this polymorphism, we used PolyPhen (Polymorphism Phenotyping), an online suite of software that predicts the possible impact of an amino-acid substitution on the structure and function of a human protein using physical and comparative considerations. ${ }^{22}$ The analysis with PolyPhen led to the conclusion that the R92Q substitution is benign. To interpret this result, one has to be aware of the still important limitations of sequence analysis software to assess the possible functional role of genetic variants; in particular, the difficulty to take into account the sequence context of the amino-acid change. This polymorphism has also been investigated by other groups and some results suggest an association with TNF-receptor-associated periodic syndrome (TRAPS). ${ }^{23-25}$

In conclusion, despite some inconsistencies, the results of three out of four studies are coherent in suggesting that the presence of the Q92 allele of TNFRSF1A may predispose to atherosclerosis and its coronary artery complications.
We believe that this hypothesis should be tested further, in particular, in relation to early MI in studies of sufficient size to account for the relatively low frequency of the Q92 allele. If the hypothesis was confirmed, it would likely have little clinical consequences, because the effect is weak and the allele raising the risk is rather infrequent. On the other hand, it would strongly suggest the causal implication of the TNF- $\alpha$ pathway in atherosclerosis development and this might have important therapeutic consequences.

\section{References}

1 Barath P, Fishbein MC, Cao J, Berenson J, Helfant RH, Forrester JS: Detection and localization of tumor necrosis factor in human atheroma. Am J Cardiol 1990; 65: 297-302.

2 Munker R, Gasson J, Ogawa M, Koeffler HP: Recombinant human TNF induces production of granulocyte-monocyte colonystimulating factor. Nature 1986; 323: 79-82.

3 Nawroth PP, Stern DM: Modulation of endothelial cell hemostatic properties by tumor necrosis factor. J Exp Med 1986; 163: 740745.

4 Pober JS, Cotran RS: Cytokines and endothelial cell biology. Physiol Rev 1990; 70: 427-451.

5 Herrmann SM, Ricard S, Nicaud V et al: Polymorphisms of the tumour necrosis factor-alpha gene, coronary heart disease and obesity. Eur J Clin Invest 1998; 28: 59-66.

6 Paleolog EM, Delasalle SA, Buurman WA, Feldmann M: Functional activities of receptors for tumor necrosis factoralpha on human vascular endothelial cells. Blood 1994; 84: 2578-2590.

7 Parra HJ, Arveiler D, Evans AE et al: A case-control study of lipoprotein particles in two populations at contrasting risk for coronary heart disease. The ECTIM Study. Arterioscler Thromb 1992; 12: 701-707.

8 Arnaud E, Barbalat V, Nicaud V et al: Polymorphisms in the $5^{\prime}$ regulatory region of the tissue factor gene and the risk of myocardial infarction and venous thromboembolism: The ECTIM and PATHROS Studies. Arterioscler Thromb Vasc Biol 2000; 20: 892-898.

9 Gariepy J, Salomon J, Denarié N et al: Sex and topographic differences in associations between large-artery wall thickness and coronary risk profile in a French working cohort - The AXA study. Arterioscl Thromb Vasc Biol 1998; 18: 584-590.

10 Herrmann SM, Whatling C, Brand E et al: Polymorphism of the human matrix Gla protein (MGP) gene, vascular calcification and myocardial infarction. Arterioscl Thromb Vasc Biol 2000; 20: 2386-2393.

11 Bonithon-Kopp C, Touboul PJ, Berr C et al: Relation of intimamedia thickness to atherosclerotic plaques in carotid arteries. The Vascular Aging (EVA) Study. Arterioscler Thromb Vasc Biol 1996; 16: $310-316$.

12 Touboul JP, Elbaz A, Koller C et al: Common carotid artery intimamedia thickness and brain infarction: the Etude du Profil Genetique de l'Infarctus Cerebral (GENIC) case-control study. The GENIC Investigators. Circulation 2000; 102: 313-318.

13 Elbaz A, Poirier O, Canaple S, Chedru F, Cambien F, Amarenco P: The association between the Val34Leu polymorphism in the factor XIII gene and brain infarction. Blood 2000; 95: 586-591.

14 Fuchs P, Strehl S, Dworzak M, Himmler A, Ambros PF: Structure of the human TNF receptor 1 (p60) gene (TNFR1) and localization to chromosome 12p13. Genomics 1992; 13: 219-224.

15 Kemper O, Wallach D: Cloning and partial characterization of the promoter for the human p55 tumor necrosis factor (TNF) receptor. Gene 1993; 134: 209-216.

16 Herrmann SM, Ricard S, Nicaud V et al: The P-selectin gene is highly polymorphic: reduced frequency of the Pro715 allele 
carriers in patients with myocardial infarction. Hum Mol Genet 1998; 7: 1277-1284.

17 Breslow NE, Day NE: The analysis of case-controls studies. Lyon, France: IARC Scientific Publications; 1980.

18 Tregouet DA, Barbaux S, Escolano S et al: Specific haplotypes of the P-selectin gene are associated with myocardial infarction. Hum Mol Genet 2002; 11: 2015-2023.

19 Rosfors S, Hallerstam S, Jensen-Urstad K, Zetterling M, Carlstrom C: Relationship between intima-media thickness in the common carotid artery and atherosclerosis in the carotid bifurcation. Stroke 1998; 29: 1378-1382.

20 Hallenbeck JM: The many faces of tumor necrosis factor in stroke. Nat Med 2002; 8: 1363-1368.

21 Allen RA, Lee EM, Roberts DH, Park BK, Pirmohamed M: Polymorphisms in the TNF-alpha and TNF-receptor genes in patients with coronary artery disease. Eur J Clin Invest 2001; 31: 843-851.
22 Ramensky V, Bork P, Sunyaev S: Human non-synonymous SNPs: server and survey. Nucleic Acids Res 2002; 30: 38943900.

23 Hull KM, Drewe E, Aksentijevich I et al: The TNF receptorassociated periodic syndrome (TRAPS): emerging concepts of an autoinflammatory disorder. Medicine (Baltimore) 2002; 81: 349 368.

24 Dode C, Andre M, Bienvenu T et al: The enlarging clinical, genetic, and population spectrum of tumor necrosis factor receptor-associated periodic syndrome. Arthritis Rheum 2002; 46: $2181-2188$.

25 Aksentijevich I, Galon J, Soares M et al: The tumor-necrosis-factor receptor-associated periodic syndrome: new mutations in TNFRSF1A, ancestral origins, genotype-phenotype studies, and evidence for further genetic heterogeneity of periodic fevers. Am J Hum Genet 2001; 69: 301-314. 